investigate relationships of lung function in early childhood with growth patterns (here considered as change in weight) before the age of five years. We use flexible longitudinal modelling methods to describe early growth trajectories, and identify factors associated with suboptimal growth.

Methods Growth measurements from diagnosis to five years were extracted from two national CF registries: UK $(n=2999$, years 2007-2015) and Canada ( $n=2690$, years 1990-2013). SITAR (super-imposition by translation and rotation) was used to model weight $(\mathrm{kg})$ over the 5 years. Output parameters were average growth curve, summaries of growth velocity and overall weight. Associations of growth and growth velocity with sex, genotype and new born screening (NBS) were investigated.

Results Most children in the UK had been diagnosed early in life (median age of diagnosis 0.06 years; inter quartile range 0.03 to 0.1 ) by NBS. 52\% were homozygous for deltaF508. Despite similar initial average weight in boys and girls, males were heavier than females over the first five years. Children homozygous for deltaF508 were lighter than other children. No tested factor was associated with velocity of weight gain. Only $10 \%$ of the Canadian children were diagnosed by NBS, and, overall, the age at diagnosis was later (median 0.17 years; inter quartile range 0.08 to 0.53 ). Over the first five years, Canadian CF children were lighter than those in the UK (figure 1). Associations of weight with sex and genotype were similar to those seen in the UK. In addition, we observed that those diagnosed by NBS were heavier than those who were not.

Conclusions In children with CF there are sex differences in weight during the first 5 years, despite similar initial weight. In Canada, NBS has a positive impact on early growth. SITAR allows exploration of growth patterns in CF patients, but time independent characteristics were not associated with velocity of growth. Statistical modelling incorporating time dependent factors (e.g., infections, treatments) are required to explain variability of growth trajectories.

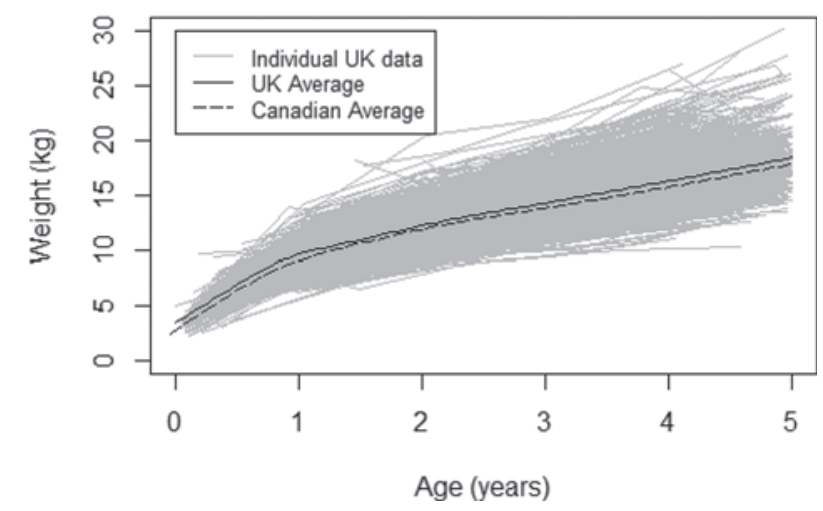

Abstract S91 Figure 1 Average growth curves for children with CF.

\section{S92 GENOMIC INVESTIGATION UNMASKS EVIDENCE OF TRANSMISSION ACROSS MYCOBACTERIUM ABSCESSUS CYSTIC FIBROSIS PATIENTS}

${ }^{1} \mathrm{~S}$ Alateah, ${ }^{2} \mathrm{C}$ Peters, ${ }^{1} \mathrm{DJ}$ Dhasmana, ${ }^{1} \mathrm{~K}$ Pettigrew, ${ }^{1} \mathrm{R}$ Fallon, ${ }^{3} \mathrm{~A}-\mathrm{L}$ Seagar, ${ }^{1} \mathrm{DJ}$ Sloan, ${ }^{3} \mathrm{IF}$ Laurenson, ${ }^{1} \mathrm{M}$ Holden, ${ }^{1} \mathrm{SH}$ Gillespie. ' School of Medicine, University of St Andrews, St Andrews, UK; ${ }^{2}$ Queen Elizabeth University Hospital, Glasgow, UK; ${ }^{3}$ Scottish Mycobacteria Reference Laboratory, Edinburgh, UK

\subsection{6/thoraxjnl-2017-210983.98}

Background Mycobacterium abscessus (Mabs) is a critical respiratory pathogen in Cystic Fibrosis (CF), with significant challenges in diagnosis and treatment. Recent whole genome sequencing (WGS) studies have challenged the assumption that acquisition in CF is primarily from soil and water systems. ${ }^{1}$ We sought to investigate potential transmission of Mabs within and between CF centres through WGS analysis of CF isolates from the Scottish Mycobacteria Reference Laboratory.

Methods 64 isolates were recovered and sequenced from CF samples positive for Mabs between 2008 and 2016. Multilocus sequence typing (MLST), and phylogenetic analysis based on core genome Single Nucleotide Polymorphisms (SNPs) was used to identify dominant circulating clones. Probable transmission clusters were defined by $<20$ SNPs between isolates, as suggested by previous studies. This work was carried out as part of a public health investigation.

Results MLST analysis of the 64 confirmed Mabs isolates demonstrated the following: 8 strain types (ST) associated with Mabs subspecies abscessus, including ST9 $(\mathrm{n}=18)$, ST24 (12), ST5 (7), ST26 (4), ST21 (3), and ST10 (2); 11 identified as subspecies massiliense associated with ST4 (4), ST6 (3), ST7 (2) and ST3 (2); 4 identified as subspecies bolletti.

Phylogenomic analysis provided higher resolution and identified significant relatedness between and within clonal complexes. The most prevalent subspecies abscessus clone was ST9, of which 16 isolates were distinguished by $<20$ SNPs, 12 of which from one hospital region. Five of the ST9 isolates were indistinguishable at the SNP level, and yet originated from different Health Board regions and different hospitals. ST5, ST24 and ST26 also contained isolates distinguished by $<20$ SNPs and originated from several geographically distinct regions.

Conclusion WGS has provided strong evidence of the circulation of dominant clones between and within CF centres across geographically diverse settings over an 8 year period. The high genomic relatedness provides strong evidence of transmission between CF patients, even though the precise mechanisms of transmission remain uncertain. Further studies are underway to understand the mechanism of transmission in this clinical context.

\section{REFERENCE}

1. Bryant JM, Grogono DM, Rodriguez-Rincon D, Everall I, Brown KP, Moreno P, Floto RA, et al. Emergence and spread of human-transmissible multidrug-resistant nontuberculous mycobacterium. Science (New York) 2016;354(6313):751-757. https://doi.org/10.1126/science.aaf8156 